\title{
Re-inventing Multimode Interference Couplers Using Subwavelength Gratings
}

\author{
A. Ortega-Moñux ${ }^{1}$, R. Halir ${ }^{1}$, A. Maese-Novo ${ }^{1}$, C. Alonso-Ramos ${ }^{1}$, L. Zavargo-Peche ${ }^{1}$, D. Pérez-Galacho ${ }^{1}$, I. \\ Molina-Fernández ${ }^{1}$, J. G. Wangüemert-Pérez ${ }^{1}$, P. Cheben ${ }^{2}$, J. H. Schmid ${ }^{2}$, J. Lapointe ${ }^{2}$, D. Xu ${ }^{2}$, and S. Janz ${ }^{2}$ \\ 1. Dpto. de Ingeniería de Comunicaciones, ETSI Telecomunicación, Universidad de Málaga, 29010, Málaga, Spain. \\ 2. National Research Council Canada, 1200 Montreal Rd., Bldg. M50, K1A OR6 Ottawa, Ontario, Canada.
}

Multimode-Interference (MMI) devices (see Fig. 1a) are fundamental building blocks in photonic integrated circuits, where they are used for power splitting and combining, optical switches and modulators, Mach-Zehnder interferometers and 90ㅇ hybrids for coherent optical receivers [1]. MMIs are based on the self-image principle, by which the guided modes of the multimode region interfere to form replicas of the input field with specific amplitude and phase relations. These relations are known to depend on i) the core/cladding refractive indexes $\left(n_{1} / n_{2}\right)$, ii) the core width (W) and length (L) of the multimode region and iii) the number, width and position of the access ports. In this work, we show that by using sub-wavelength structures within an MMI, the self-imaging properties can be significantly altered, leading to ultra-short or ultrabroadband devices.

Subwavelength gratings (SWGs) are structures with a pitch below the Bragg condition, so that they behave as an effective medium [2]. This means that in Silicon-on-Insulator (SOI) technology, equivalent refractive indexes between those of the core $(\mathrm{Si})$ and the cladding (e.g. $\mathrm{SiO}_{2}$ or $\mathrm{SU}-8$ ) can be obtained tuning the SWG pitch and duty cycle. This "refractiveindex-engineering" has already been successfully applied to a variety of integrated devices $[3,4]$. The dispersion properties of SWGs have only very recently been explored [5].

Here we use the concept of refractive index engineering to propose and experimentally demonstrate a reduced size, slotted $2 \times 2 \mathrm{MMI}$ coupler. The key point of this device is the design of a longitudinal SWG slot placed at the center of the multimode region (Fig. 1b). When properly designed, this slot changes the propagation constants of the even modes without affecting the odd modes. As a result the length of the device is halved without degrading its performance: Fig. 1 (c) shows that, in a back-to-back configuration, the shortened device even exhibits a slightly better measured extinction ratio than the conventional device. We also present an ultra-broadband $2 \times 2 \mathrm{MMI}$ SWG coupler (Fig. 1d) which is based on SWG dispersion engineering. The tapered SWG structures at the input and output provide low-loss and low-reflection transitions between conventional silicon-wire waveguides and the SWG multimode region. The SWG multimode region is designed to achieve a nearly wavelength independent self-imaging length. The device exhibits a bandwidth of $450 \mathrm{~nm}$ (1260nm-1675nm), which is five times higher than conventional MMIs. In this range, the insertion loss, power imbalance and $\mathrm{MMI}$ phase error, as simulated with 3D FDTD, are below $1 \mathrm{~dB}, 0.6 \mathrm{~dB}$ and $3 \circ$, respectively.
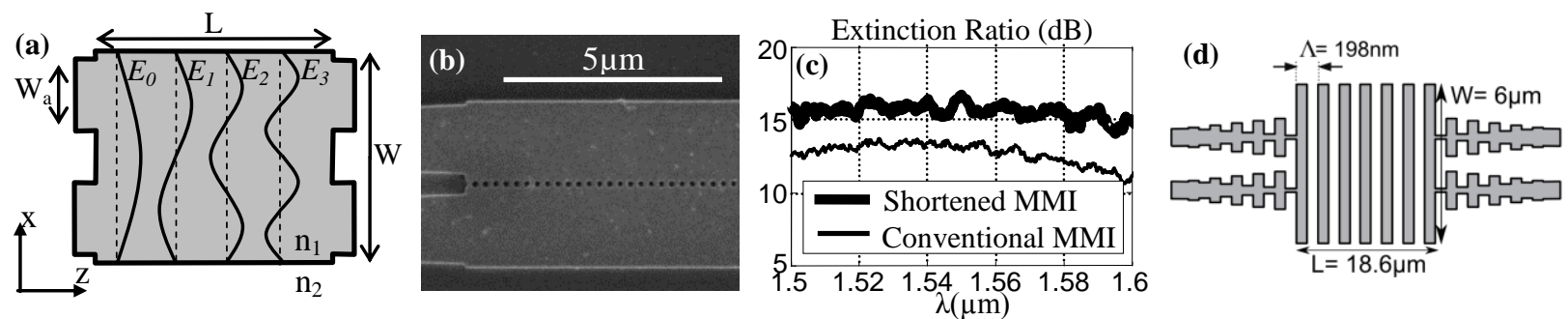

Fig. 1 (a) Geometry of a conventional 2x2 MMI coupler. $E_{0}, E_{1}, E_{2}$ and $E_{3}$ are the field profiles of the four lowest order modes. (b) Scanning Electron Microscope Image of the reduced size slotted 2x2 MMI coupler. (c) Measured extinction ratio of fabricated back-to-back MMI test structures. Device footprints are: Shortened $3.5 \mu \mathrm{m}$ x $23 \mu \mathrm{m}$ and Conventional $3.5 \mu \mathrm{m} \times 47 \mu \mathrm{m}$. (d) Geometry of the ultra-wideband $2 \times 2 \mathrm{MMI}$ coupler with SWG multimode region.

\section{References}

[1] L. B. Soldano and E. C. M. Pennings, “Optical multi-mode interference devices based on self-imaging: Principles and applications,” J. Lightw. Technol. 13, 615 (1995).

[2] S. M. Rytov, "The electromagnetic properties of finely layered medium," Sov. Phys. JETP, 2, 466-475, 1956.

[3] P. Cheben, P. Bock, J. Schmid, J. Lapointe, S. Janz, D. Xu, A. Densmore, A. Delage, B. Lamontagne, and T. Hall, "Refractive index engineering with subwavelength gratings for efficient microphotonic couplers and planar waveguide multiplexers," Opt. Lett. 35, 2526 (2010).

[4] R. Halir, P. Cheben, J. H. Schmid, R. Ma, D. Bedard, S. Janz, D.-X. Xu, A. Densmore, J. Lapointe, and I. Molina-Fernández, "Continuously apodized fiber-to-chip surface grating coupler with refractive index engineered subwavelength structure," Opt. Lett. 35, 3243 (2010).

[5] R. Halir, A. Maese-Novo, A. Ortega-Moñux, I. Molina-Fernández, J. G.Wangüemert-Pérez, P. Cheben, D.-X. Xu, J. H. Schmid, and S. Janz, "Colorless directional coupler with dispersion e ngineered sub-wavelength structure," Opt. Express 12, 13470 (2012).

Acknowledgements. This work was supported in part by the Spanish Ministerio de Ciencia (project TEC2009-10152), a Formación del Profesorado Universitario scholarship (AP-2006-03355), the European Mirthe project (FP7-2010-257980) and "Universidad de Málaga. Campus de Excelencia Internacional Andalucía Tech" 